\title{
mHealth: Where Is the Potential for Aiding Informal Caregivers?
}

\author{
Meghan BRADWAY ${ }^{\mathrm{a}, 1}$, Ashenafi Zebene WOLDAREGAY ${ }^{\mathrm{b}}$, David-Zacharie ISSOM ${ }^{\mathrm{c}}$, \\ Gerit PFUHL $^{\mathrm{d}}$, Gunnar HARTVIGSEN ${ }^{\mathrm{b}}$, Eirik ARSAND $^{\mathrm{a}, \mathrm{b}}$ and André HENRIKSEN ${ }^{\mathrm{b}}$ \\ ${ }^{a}$ Norwegian Centre for E-Health Research, University Hospital of North Norway \\ ${ }^{\mathrm{b}}$ Department of Computer Science, UiT The Arctic University of Norway \\ ${ }^{c}$ Geneva University Hospitals, Division of Medical Information Sciences, Switzerland \\ ${ }^{d}$ Department of Psychology, UiT The Arctic University of Norway
}

\begin{abstract}
The health and well-being of informal caregivers often take a backseat to those that they care for. While systems, technologies, and services that provide care and support for those with chronic illnesses are established and continuously improved, those that support informal caregivers are less explored. An international survey about motivations to use mHealth technologies was posted to online platforms related to chronic illnesses. We focused on responses regarding the facilitators and challenges of achieving health goals, including the use of mHealth technologies, for the subgroup who identified as "Caregivers". Findings indicate that mHealth technology is not yet the most important motivational factor for achieving health goals in this group, but greater future potential is suggested.
\end{abstract}

Keywords. Caretakers, chronic, diabetes, sickle cell, motivation, self-management

\section{Introduction}

Informal caregivers play a crucial role in the complex network surrounding the care of those with chronic illnesses. These caregivers are defined as family members, friends, or other community members who provide practical, physical, and psychological support for those with chronic illnesses. Formal healthcare providers receive years of training, medical and administrative staff, technologies tailored to their needs, protocols, and financial support - support that informal carers are often not provided [5]. As the demand on healthcare systems continues to surpass the supply of medical resources, this excess demand spills over into the responsibility of informal carers [3].

The 2016 European Quality Of Life Survey (EQLS) revealed that the prevalence of informal carers varies greatly by country (10\%-33\%) [11]. The EQLS also highlighted that $27 \%$ of working caregivers and $41 \%$ of unemployed caregivers reported being "In fair or bad health" [11]. Caregiver burden is characterized by both physical and psychological symptoms and disorders associated with, or resulting from, the stress of providing $20+$ hours of care per week - varying greatly between diagnosis groups of those whom they care for [1]. According to the Center for disease control (CDC), Informal caregivers themselves are at risk for developing chronic illnesses such as

\footnotetext{
${ }^{1}$ Meghan Bradway, University Hospital of North Norway's Norwegian Centre for E-health Research, Sykehusvegen 21, 9019 Tromsø, Norway, E-mail: Meghan.Bradway@ehealthresearch.no
} 
depressive disorders, diabetes, cancer, coronary heart disease, with approximately $41 \%$ of caregivers reporting being diagnosed with $2+$ chronic illnesses [2]. While little formal support or interventions are designed to aid or understand the needs of caregivers, mobile and digital health interventions have the potential to support one's own health selfmanagement as well as their tasks as caregivers [9]. Caregiver motivation is, and only recently, directed at those whom they care for [4]. We aim to contribute to this foundational knowledge by introducing concepts that have yet to be explored at length to contribute to the design of further research.

In this paper, we present selected results of and international cross-sectional online survey, "Motivation in mobile health", regarding personal health, motivational factors, and use of mHealth technologies for self-management with the focus on self-reported caregivers. The purpose of focusing on this subgroup is to highlight the unique needs of those who are caretakers and those who are caretakers with chronic illnesses, and to put these fourth as potential focus areas for mHealth development.

\section{Methods}

The online survey was distributed on social media forums related to sickle cell disease (SCD) or diabetes $(\mathrm{n}=9$ online platforms total). We used results from 16 in-person interviews to derive questions for the survey [10]. Announcements in either English, Norwegian, or French language called for respondents to answer questions about what motivates them to self-manage their own health or illness. Questions $(n=38)$ were separated into seven categories with the following headings: 1) background and health goal questions, 2) use of wearables and sensors, 3) use of mobile apps, 4) data-logging, 5) data sharing and data integration, 6) social media and entertainment factors, and 7) demographic questions including age, gender, chronic disease diagnosis. Questions about "with who do you prefer to discuss health issue", and caretaker status were added to this last section of the survey after the first 257 respondents answered. The online survey was open for data collection between November 2018 and March 2020. Primary results comparing those with and without chronic illnesses have been previously published [10] and further details about the questionnaire are available at DataverseNO [6].

While caregivers were not the primary target group for this survey, questions regarding whether a person was a caregiver, whom they provided care for, and whether they had a chronic illness themselves were placed at the end of the survey. In doing so, we were able to gather the same and comparable information on all respondents, which also limited the bias of such relationships on their previous responses regarding the perception of their own health, access to quality health information, use of mHealth technologies. A request for ethical approval was reviewed by Veronica Sørensen at the Regional Ethics Committee (REK) and found to be exempt from their purview (ref. 2017/562).

\section{Results}

A total of 539 people responded to the online survey that included questions about being a caregiver, 72 of whom reported being caregivers $(n=46$ had a chronic disease 
themselves, $n=24$ who had no chronic disease, and $n=2$ who left this question blank). These respondents ( $n=46$ female, mean age 39.8 years; $n=20$ male, mean age 42.8 years, $\mathrm{n}=6$ did not disclose their gender, mean age 28.6 years) represented $\mathrm{n}=25$ countries $(\mathrm{n}=30$ USA, $n=11$ Norway, $n=8$ UK, $n=2$ Australia and $n=1$ for remaining countries). Caregivers reported caring for those with $\operatorname{SCD}(n=9)$, diabetes (type I: $n=9$; type II: $n=4$ ), and other chronic illnesses $(\mathrm{n}=40)$. Results are divided into two groups, i.e. Caregiver only or a Caregiver with chronic illness. Table 1 describes caregivers' primary health goals, given the following options: to Avoid illness, Improve illness, Recover from existing illness, or Other health goal.

Table 1. Caregivers' primary health goals

\begin{tabular}{ccc|c}
\hline Primary health goal & Caregiver only $(\mathbf{n = 2 4})$ & $\begin{array}{c}\text { Caregiver with chronic } \\
\text { illness }(\mathbf{n = 4 6})\end{array}$ & Caregivers $(\mathbf{n}=\mathbf{7 2}) *^{*}$ \\
\hline Avoid & $65 \%$ & $41 \%$ & $50 \%$ \\
Improve & $23 \%$ & $37 \%$ & $32 \%$ \\
Recover & $8 \%$ & $17 \%$ & $14 \%$ \\
Other & $4 \%$ & $4 \%$ & $4 \%$ \\
\hline
\end{tabular}

*Includes two caregivers who left the question "do you have a chronic illness" blank.

As can be seen in Figure 1 the two groups rated the importance of the eight motivational factors very similarly (no group difference, $\mathrm{F}<1, \mathrm{p}=.61$, no interaction, $\mathrm{F}<1$, $\mathrm{p}=.81$ ). Apart from the access to Advanced/relevant technology all other goals are rated as very important (this difference between factors was significant, $\mathrm{F}(4.373,240.516)=8.33, \mathrm{p}<.001)$.

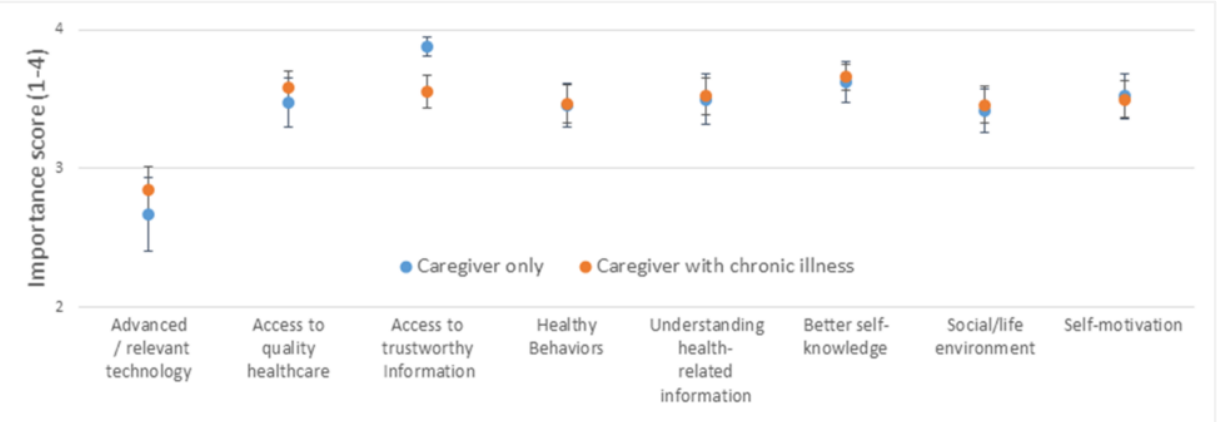

Figure 1. Importance of factors to achieve one's primary health goal (multiple-choice). Error bars denote SEM

As shown in Figure 2, the groups Caregiver only and Caregiver with chronic illness demonstrated similar distributions in the category "Challenges of achieving one's primary health goal" related to their experienced Limited options for technology, Lack of time, and Lack of health knowledge, yet differed in reported challenges of Motivation (38\% and $28 \%$ respectively), Lack of social support ( $0 \%$ and $9 \%$ respectively), Lack of access to quality healthcare (17\% and $28 \%$ respectively), and Other $(13 \%$ and $5 \%$ respectively). 


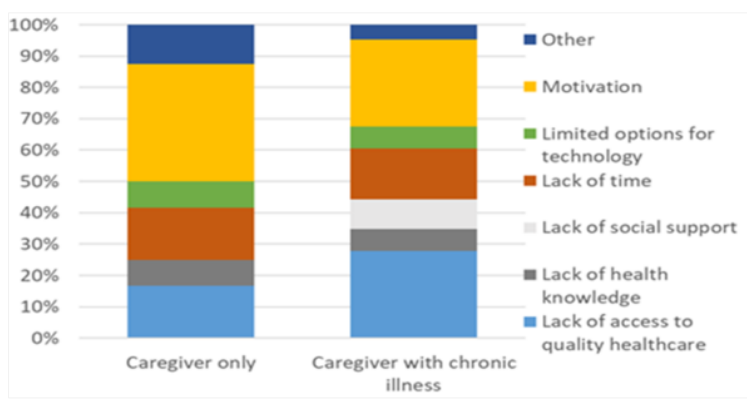

Figure 2. The greatest challenge to achieving one's primary health goal, by group

Of all caregivers, $65 \%$ reported using one mHealth technology, $15 \%$ used two, $14 \%$ used three, and 5\% used four or more mHealth technologies. Table 2 shows the distribution of which technologies caregivers use. Most of those in the Caregiver only group used Sensors (integrated into the smartphone, e.g. geolocation, stress tracker) and Other devices, whereas those in the Caregiver with chronic illness group used mostly Medical devices and Apps.

Table 2. Percentage of caregivers who use mobile health technologies, by group

\begin{tabular}{rcc|c}
\hline Technology used & Caregiver only $(\mathbf{n = 2 4 )}$ & $\begin{array}{c}\text { Caregiver with chronic } \\
\text { illness }(\mathbf{n = 4 6})\end{array}$ & Caregivers $(\mathbf{n}=\mathbf{7 2}) *^{*}$ \\
\hline Sensors & $38 \%$ & $26 \%$ & $29 \%$ \\
Activity trackers & $33 \%$ & $33 \%$ & $32 \%$ \\
Apps & $29 \%$ & $39 \%$ & $35 \%$ \\
Medical devices & $13 \%$ & $46 \%$ & $35 \%$ \\
Other devices & $42 \%$ & $22 \%$ & $29 \%$ \\
\hline
\end{tabular}

*Includes two caregivers who yet left the question "do you have a chronic illness" blank.

Table 3 illustrates the different reasons respondents had for collecting data using their mHealth technologies. The biggest differences were related to the reasons of Competition and Other, which were favored by those in the Caregiver only group, and Tracking progress, which was favored by the Caregiver with chronic illness group.

Table 3. Caregivers' reasons for using mHealth technology to gather data, by group

\begin{tabular}{rrr|r}
\hline \multicolumn{1}{c}{ Motivations } & Caregiver only (n=24) & $\begin{array}{c}\text { Caregiver with chronic } \\
\text { illness }(\mathbf{n = 4 6})\end{array}$ & Caregivers $(\mathbf{n}=\mathbf{7 2}) *^{*}$ \\
\hline To track progress & $67 \%$ & $78 \%$ & $74 \%$ \\
To share data & $25 \%$ & $26 \%$ & $25 \%$ \\
Competition & $17 \%$ & $4 \%$ & $8 \%$ \\
To learn & $71 \%$ & $76 \%$ & $74 \%$ \\
To receive feedback & $29 \%$ & $35 \%$ & $32 \%$ \\
Other & $17 \%$ & $2 \%$ & $7 \%$ \\
\hline
\end{tabular}

*Includes two caregivers who yet left the question "do you have a chronic illness" blank.

The two groups, Caregiver only and Caregiver with chronic illness, also reported preferring to seek health information from the following sources: healthcare providers ( $50 \%$ and $46 \%$ respectively), Carers ( $0 \%$ and $4 \%$ respectively), or online (33\% and $39 \%$ respectively). 


\section{Discussion}

Caregivers represent an unmet group of potential users of mobile and digital health technologies with unique and diverse needs. However, our data (Figure 1) shows that technology is not yet a particularly motivating or facilitating factor when it comes to achieving their health goals. The fact that $65 \%$ of caregivers reported using one mHealth technology, and that both groups reported collecting data to track their progress and learn about their health, suggests there is potential for these technologies to become a greater factor in achieving health goals, e.g. by supporting other factors which they already deem important. While overall caregivers primarily wish to avoid illness, those who are only caregivers favor avoiding, whereas those who were caregivers with a chronic illness favor improving or recovering from existing illness. Other differences seen throughout the results could be potentially significant in larger sample sizes and should be explored in future research.

Limitations: the following factors contributed to a relatively small sample size of caregivers: i) the language of the recruitment analysis, i.e. describing health in terms of "illness" or "disease", may have deterred those who otherwise would have responded to the more general term "health", ii) the survey did not specifically target caregivers, iii) the recruitment platforms were primarily related to diabetes and sickle cell disease, and iv) the survey was only available in English, Norwegian, and French.

Considerations for future research: Because caregivers can perceive their duties as positive and fulfilling, stressful and burdensome, or anywhere in between, a person's attitudes can highly influence their capacity and willingness to take care of themselves. While static information about how to care for oneself while caring for others is available, technology has the potential to make this more accessible [7]. While unexplored in this survey, it has been noted by others that a person's country of residence, their associated medical systems, and cultural factors also affect their perception of their role as a caregiver, as well as their personal capacity and practical ability to additionally care for themselves [8]. By targeting the unique needs of caregivers, when caring for themselves and others, mHealth technologies also have the potential to help that person -manage their own health. Therefore, future research about the needs, priorities, and challenges of caregivers and the design and development of mHealth technologies should consider these factors.

\section{Conclusion}

As anyone who is ever flown in a plane knows, safety presentations instruct us to help ourselves by putting on our own oxygen mask first before assisting others. After all, we are no good to anybody if we are incapacitated. Therefore, it is just as important to understand the needs and risks of caregivers, a crucial group of stakeholders related to not only their ability to care for their friends and family with chronic illnesses but also themselves.

This survey has gathered a diverse set of information. We encourage our fellow researchers to contact us to discuss and collaborate on future analysis. 


\section{References}

[1] Adelman RD, Tmanova LL, Delgado D, Dion S, Lachs MS. Caregiver burden: a clinical review. JAMA. 2014 Mar 12;311(10):1052-60.

[2] Centers for Disease Control and Prevention, Caregiving for family and friends - a public health issue. 2018. Available at: https:/www.cdc.gov/aging/caregiving/caregiver-brief.html. Accessed 2020.

[3] de Jong L, Plöthner M, Stahmeyer JT, Eberhard S, Zeidler J, Damm K. Informal and formal care preferences and expected willingness of providing elderly care in Germany: protocol for a mixed-methods study. BMJ Open. 2019 Jan 15;9(1):e023253.

[4] Dombestein H, Norheim A, Lunde Husebø AM. Understanding informal caregivers' motivation from the perspective of self-determination theory: an integrative review. Scand J Caring Sci. 2020 Jun;34(2):267279.

[5] Hengelaar AH, van Hartingsveldt M, Wittenberg Y, van Etten-Jamaludin F, Kwekkeboom R, Satink T. Exploring the collaboration between formal and informal care from the professional perspective-A thematic synthesis. Health Soc Care Community. 2018 Jul;26(4):474-85.

[6] Woldaregay AZ, Henriksen A, Issom DZ, Pfuhl G, Sato K, Richard A, Lovis C, Årsand E, Rochat J, Hartvigsen G. User Expectations and Willingness to Share Self-Collected Health Data. Stud Health Technol Inform. 2020 Jun 16;270:894-898.

[7] Sullivan AB, Miller D. Who is Taking Care of the Caregiver? J Patient Exp. 2015 May;2(1):7-12.

[8] Verbakel E. How to understand informal caregiving patterns in Europe? The role of formal long-term care provisions and family care norms. Scand J Public Health. 2018 Jun;46(4):436-447.

[9] Wasilewski MB, Stinson JN, Cameron JI. Web-based health interventions for family caregivers of elderly individuals: A Scoping Review. Int J Med Inform. 2017 Jul;103:109-138.

[10] Woldaregay AZ, Issom DZ, Henriksen A, Marttila H, Mikalsen M, Pfuhl G, Sato K, Lovis C, Hartvigsen G. Motivational Factors for User Engagement with mHealth Apps. Stud Health Technol Inform. 2018;249:151-57.

[11] Zigante, V., Informal care in Europe, Exploring Formalisation, Availability and Quality, EC (2018), 438. Available at: https://ec.europa.eu/social/main.jsp?catId=738\&langId=en\&pubId $=8106 \&$ furtherPubs $=$ yes. 